\title{
Competencies for international nursing research in a wikinomics future
}

\author{
Mary Anne Schultz, Benson Yeung, Jane Frances Hook \\ School of Nursing, California State University, Los Angeles, United States
}

Correspondence: Mary Anne Schultz. Address: School of Nursing, California State University, Los Angeles, United States. Email: mschult2@calstatela.edu

Received: December 31, 2011

Accepted: May 7, 2012

Published: November 1, 2012

DOI : $10.5430 /$ jnep.v2n4p178

URL: http://dx.doi.org/10.5430/jnep.v2n4p178

\section{Abstract}

Conducting nursing research in the international arena provides unique challenges previously unseen in the pre-Web 2.0 world. Facilitated by the combined use of cloud and tablet computing, the means to address such challenges are identified by the authors, based on their experiences as international consultants in informatics, multinational research, and strategy in China and Southeast Asia. Using a framework on Wikinomics by Tapscott and Williams, the authors discuss how a new approach to research is made possible using the "weapons of mass collaboration” modeled by Wikipedia ${ }^{\circledR}$, Facebook ${ }^{\circledR}$, and YouTube. Research tools such as Skype ${ }^{\circledR}$, Tape-a-Talk ${ }^{\circledR}$, Google Cloud ${ }^{\circledR}$ and voice-to-text applications are described.

\section{Key words}

Competencies, Wikinomics, International research

\section{I ntroduction}

Based on the experiences of the authors in China and Southeast Asia, specific aspects of data collection and data retrieval are discussed in the context of e-Science and a Wikinomics framework. Discussion of specific tools such as voice-to-text applications and data display models illustrate how "weapons of mass collaboration" facilitate data-driven aspects of the research process within the new model. These are presented as the new competencies for the nurse researcher in a Wikinomics framework.

\subsection{Wikinomics: How mass collaboration changes everything}

In a seminal work, Tapscott and Williams ${ }^{[1]}$ explain how individuals and organizations are harnessing principles of mass collaboration to drive innovation in their workplaces, communities, and organizations. They claim that, through principles such as openness and peering, or peer production, the triad of human skill, ingenuity, and intelligence will become more effective than anything that we have witnessed before. The application of these principles by leaders in organizations will result in a product or service that is "quicker, better, cheaper" as well as of lasting value ${ }^{[1]}$.

The first characteristic, openness, involves opening up infrastructures and intellectual property to the market, as have Amazon and Google, as a means to hasten innovation. More than a state of mind, openness generally refers to a culture of 
connectivity fostered by many of the Web 2.0 tools. Regarded by O'Reilly ${ }^{[2]}$ as "an architecture of participation", openness involves content sharing and the removal of limitations inherent in analogue systems, expanding the type of things that people can share. Wikipedia ${ }^{\circledR}$, the free encyclopedia that anyone can edit, is the most salient example of how user-generated content results in an open-source repository of the collective knowledge of networked experts worldwide. Similarly, today's researcher casually comments on another researcher's scholarly projects on Twitter ${ }^{\circledR}$, collaborates on a paper while working in Google Docs, shares his or her presentation slides on Slideshare, and eventually submits his or her papers to open access journals, where they are reviewed and published within two months and read by as many people worldwide as have an interest in the topic. This scenario of the individual scholar's network as a means of distribution has much further reach than do traditional distribution channels, such as publishers, libraries, and book retailers, which are limited by the restriction of a subscription basis.

In traditional networking, collaborating with a select few colleagues inside and outside the scholar's home institution is limited. The nurse researcher can supplement the traditional research process by adopting principles of mass collaboration, which enable the nurse researcher to employ intelligence gathered on a worldwide basis. In subsequent sections, the authors will show how tablet computing provides rapid mobile access to documents, sites, and humans "in the cloud", which allows the researcher to make the transition from owning knowledge to sharing it.

\subsection{Every nurse researcher an informaticist: The transformative power of eresearch}

Scholarship is undergoing transformation in amazing ways. Many suggest that the very essence of the research enterprise is changing. Largely due to the enabling power of the Internet, the everyday procedures and practices of traditional research are undergoing a period of rapid change from traditional university-centric interdisciplinary team investigations to large-scale mass collaborative efforts. Some of the overall changes, as abstracted from Jankowski ${ }^{[3]}$ are:

- Reliance on networked virtual structures for conducting research increasingly reliant upon distance collaboration among researchers, often internationally;

- Development of Internet-based tools that facilitate many phases of the research process, including communication, research management, data collection, and analysis, as well as e-publishing;

- Experimentation with new ways to visualize data, such as network and hyperlink analysis and multimedia and dynamic representations;

As every nurse researcher acquires informatics competencies, e-Science will refer to the large-scale science that will increasingly be carried out through distributed global collaborations enabled by the Internet ${ }^{[4]}$. Typically, the Wikinomic feature of mass collaboration in such scientific enterprises will involve large data collections such as state or national hospital mortality data, large-scale computing resources, and high-performance visualization of data for the individual user-scientist ${ }^{[5]}$. This type of collaboration, accomplished by the technique of crowdsourcing, enables the modern researcher to realize the potential of a distributed model of gathering user input.

\section{Data collection, retrieval, and analysis}

A variety of tablet-compatible tools are available to the researcher who wishes to enter or record data, modify a data set, and visualize the data as an investigation is underway. This capacity is particularly important for gathering data in China and the ASEAN (Association of Southeast Asian Nations) countries, such as Vietnam or Thailand, where a national technology adoption policy is weak or absent ${ }^{[6]}$.

For audio data collection such as one-to-one interviews, Skype ${ }^{\circledR}$ add-ons such as Kish Kish ${ }^{\circledR}$ SAM records conversations automatically when the party being called answers. Voice over Internet Protocol (VoIP) converts analogue voice signals 
into digital data packets, thus supporting a two-way real-time conversation through an Internet Protocol (IP). Hot Recorder for VoIP, a popular plug-in for the PC, is also used for two-party interview methods. These tools, compatible with iPad or Droid tablets, are suitable for qualitative research in which data are coded prior to thematic analysis. Tape-a-Talk ${ }^{\circledR}$, a high-quality voice-to-text app designed for simplicity, can record voice notes as well as substantive conversations or conference calls. Even researchers on a skeletal budget should consider these tools as a foundational means of data collection. With a combination of these tools and a voice-to-text app such as Dragon NaturallySpeaking ${ }^{\circledR}$, the need for a transcriptionist is obviated. Records obtained are exported as wav or mp3 files, which are easily stored in and retrieved from a cloud and are accessible to the research team from any location worldwide. A brief description of Dragon NaturallySpeaking ${ }^{\circledR}$ and Tape-a-Talk ${ }^{\circledR}$ ensues focusing on the use of these tools by the mobile nurse researcher.

The nurse researcher wishing to conduct traditional face-to-face (F2F) or virtual interviews could be interested in Dragon NaturallySpeaking 9.0®. This software can be used with the SONY ICD SX-57 Digital voice recorder for those more traditionally grounded or through iPad and Android apps. Consider Figure 1 which illustrates a logical starting point where the user merely pushes the 'tap and talk' button to begin. The user then speaks clearly and simply (somewhat robotically) into the device, saying "correct" when the app chooses the wrong word and selecting the correct one as prompted. Tap again to stop the recording and begin the text conversion. As the text is shown in the main window, the first part of the dictation becomes the name of this note. The mobile nurse researcher who has subjects using pseudonyms will want to begin the session by recording that pseudonym at that moment as there is no way to rename the file once it is begun.

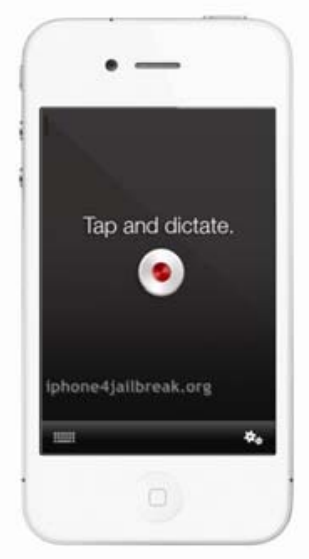

Figure 1. Dragon NaturallySpeaking Interface

Correcting the text for accuracy will be necessary yet this step can logically be done in the early phase of thematic analysis. As the text is corrected, press the down arrow in the top right corner of the window to view options for cutting and copying the text, adding it to the body of an email, posting it to Facebook ${ }^{\circledR}$ or Twitter ${ }^{\circledR}$, or placing it in cloud storage.

AudioNote ${ }^{\circledR}$, simpler voice-record software, is an enhanced note-taking system in which added handwritten or typed notes create tags for queries and audio placeholders. Sometimes confused as a voice-to-text app, AudioNote ${ }^{\circledR}$, is more useful for a student to record lectures. Dragon NaturallySpeaking $9.0{ }^{\circledR}$, instead, is a speaker-dependent, multi-party, small-vocabulary form of intelligence. It is not appropriate for recording of group processes in which a larger number of participants is expected. For that type of encounter, technology necessary would be of a speaker-independent, multi-party, large-vocabulary type and exists now only in the development phase.

The qualitative researcher interested in just audio recordings, without text-added features, can choose from a number of one-touch recorder products, the simplest of which is the Tape-a-Talk app. Commonly used by those wishing to record notes, interviews or lectures, an exportable space-saving 3gp file is created. It is also possible to create an enhanced wav 
file, which allows use of the "suspend" and "resume" features. Built-in sharing to Dropbox, email or other third-party services such as clouds is supported. Custom file names can and should be systematically created at the point of data gathering. Note at the bottom of Figure 2 below where the process begins by merely pressing the red record button and using the pause button next to it as needed. The disadvantage to Tape-a-Talk is its uninviting interface.

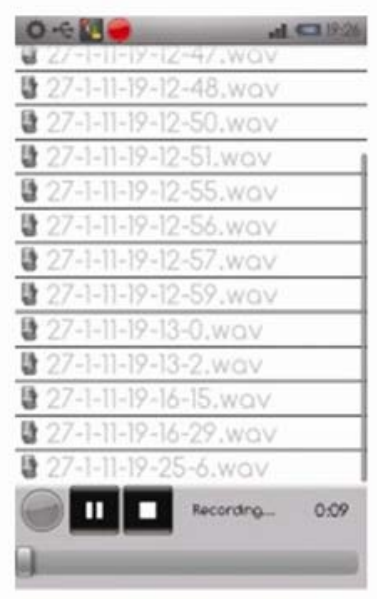

Figure 2. Tape-a-Talk Interface

The quantitative researcher who performs data collection while in residence can begin data manipulation, including diagnostics, at the point of data collection. With the use of SVG Interactive figures, or scalable vector graphics, a file format allows a two-dimensional image to be displayed in XML pages on the Web. Interactive SVG can be used to display data in multiple ways and at multiple levels, which is an advantage over traditional, static two-dimensional data display, especially when working with large data sets. Different from JPEG or GIF images that are bitmapped and always remain a specified size, SVG figures are scalable to the size of the viewing window and will adjust to the window's size and resolution. Figure 3, an easily recognizable image from Wikimedia Commons, is a side-by-side comparison of bitmap versus vector images. Simply put, the former is a fixed set of static dots while the latter is a dynamic set of fixed shapes.

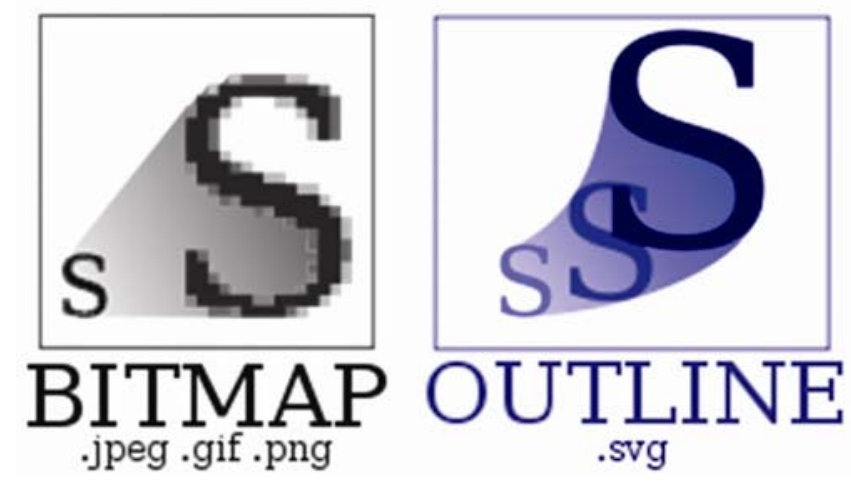

Figure 3. The Difference between Bitmap and Vector Images

A nurse researcher who explores the influence of volume of cases, staffing variables, profit status, and other structural features of hospitals on complication rates usually has a very large database from which to abstract data. The Kroch Research Group ${ }^{[7]}$ used MedPAR data, abstracted from a national data bank of nearly 3,000 hospitals, to explore aspects of U.S. hospital performance on select quality measures, including complication rates. With data sets and data display models such as SVG, the researcher can quickly visualize important aspects of data analysis such as the distribution of the 
outcome variable, complications. Important in the data cleaning procedures, as more descriptors of the organization are understood, the researcher can further diagnose and treat missing cases, data redundancy, multicollinearity, heteroscedasticity, and other statistical conundrums. In attempting replication of such a study in developing countries where there are no such large databases, the researcher can use data and information acquired while in residence to risk-adjust the outcome variable of interest as he or she explores comparability of hospitals for benchmarking.

\subsection{Brief background on cloud computing}

In 2007, “cloud computing” was introduced and represented a computing paradigm shift aimed at providing end users with the opportunity to store, access, share, and consume information via the Internet ${ }^{[8]}$. Concepts of computing via the “cloud," a metaphor for the Internet, date back to the late 1950s, when the famous Canadian researcher Douglas Parkhill ${ }^{\text {[8] }}$ described characteristics of modern-day clouds. The term, as commonly used today, means a web-based document sharing and storage system.

In addition to the ability to send and receive information over the "cloud," end users also can access information, both public and private, via devices such as laptop computers, smart phones, or tablets in real time and in a synchronized manner ${ }^{[9]}$. Other advantages of cloud computing include convenient data access points, flexible and expandable (elastic) storage locations ${ }^{[10]}$, multi-tenancy resource allocation, reduced overall infrastructure costs and maintenance, and ease of use.

Nurse faculty are already working "in the cloud" but, like many other end-users, may not be aware that they are. A good example of "being in the cloud," while not being aware of it, is the use of dashboards such as TaskStream ${ }^{\circledR}$, where faculty and staff of one institution merge, and then share, data on each student with nursing leaders in the institutions in which they affiliate. TaskStream ${ }^{\circledR}$ is one of many new Web-based accountability software management systems that make the onboarding of students who do fieldwork relatively seamless, while maintaining the integrity of the verification process.

Research on the use of cloud computing in nursing and medicine is scarce. A significant opportunity exists for researchers to extend their sphere of investigative interests to subjects in developing nations through the use of cloud computing. For example, in a study conducted to evaluate the feasibility and potential impact of an interactive voice response (IVR) program using a cloud-computing model to improve diabetes management in Honduras, Piette et al. ${ }^{[11]}$ found that cloud computing was a feasible strategy for providing IVR. In particular, they found better disease management, such as better glycemic control, in those subjects who completed a higher percentage of their IVR diabetes management calls. Thus, a nurse researcher whose population of interest is adult diabetics could attempt to replicate interventions for diabetes management in U.S. adult diabetics with diabetics in developing nations.

With relatively few studies on the use of cloud computing in healthcare, it may be difficult for the traditional nurse researcher to conceptualize how to incorporate cloud computing into a program of research. The authors of this article, however, had the opportunity to explore some functions of cloud computing, utilizing their own international nursing research collaboration model in Vietnam, Thailand, and China over the last three years. Through these experiences, the authors determined that there are many opportunities in international research for similarly-interested investigators. A brief description of select features of Google Cloud follows. This is designed to show the mobile nurse researcher a venue for documents and communication that is likely to be foundational to the work of the project. The features of the site should be explored by the mobile nurse researcher prior to field work abroad.

When the terms "cloud" or "cloud computing" arise, many nursing professionals envision some complex online environment with mysterious-sounding features such as exporting, Google Voice SMS to email, and transcription. But in fact, one basic uncluttered feature of Google Cloud computing is document sharing. Figure 4 below is a screenshot of “Google Docs”, Google’s SaaS (software-as-service) Office Suite. 


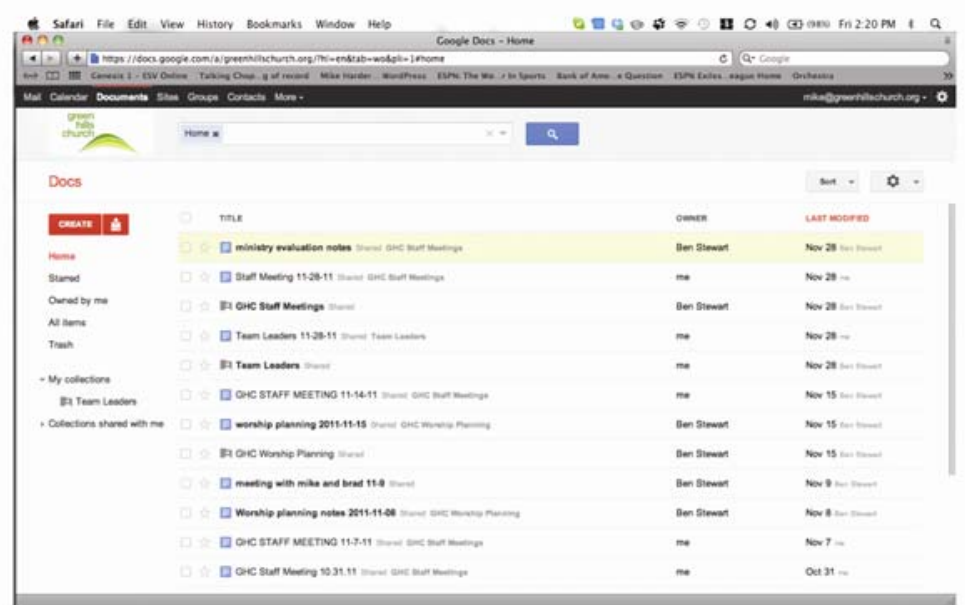

Figure 4. Google Docs Screenshot

Documents, spreadsheets, and presentations can be created, stored and shared selectively on Google Docs amongst a set of professionals working on a joint project. As seen in the screenshot, on the left is a list of document titles ("Title") and, on the near right, a list of those who have access to them ("Owner"). These "clouds" or "collections" are files with a common purpose for the owner and those users who the owner designates as users who may view and/or edit the documents. A convenient feature of Google Docs is the revision history of each document ("Last Modified"). Another feature of Google Docs is the ability multiple users have to edit a document in real time. The ability to mass collaborate across geographic areas, users and time zones is emblematic of cloud computing.

\subsection{Limitations of cloud computing}

Although cloud computing offers many benefits (simplicity, low cost, capacity, security), there are significant limitations. The major limitations include dependence on uninterrupted high-speed Internet access, some challenges with privacy and security, lack of applications for field research, and issues with useability. Each is discussed below.

When conducting research abroad, one of the major limitations of cloud computing, especially in developing countries, is the scattered availability of uninterrupted high-speed Internet access. Due to faulty Internet structure and/or Internet firewalls installed by authoritarian governments, it is less expensive and easier to navigate the Web via wireless hot spots in the United States and other western nations. Additionally, social network services such as Google ${ }^{\circledR}$, YouTube ${ }^{\circledR}$, and Twitter ${ }^{\circledR}$ are often not sanctioned by local government, leaving researchers and other end users with blocked, compromised, or absent sites or content.

Privacy is another concern when conducting research abroad. Sensitive information related to subjects' demographics, health, or financial backgrounds are uploaded and stored in the cloud servers. Because content ownership rights and the rights to access data by the cloud service providers (administrators or IT personnel) are still not absolute, the ultimate privacy of research subjects cannot be assured. Although most cloud service providers would ensure that their servers are kept free from computer infections, malwares, or attacks, the potential for hacking still exists. To ensure proper data security so that unauthorized users cannot gain access to the information, researchers should use data encryption prior to uploading all sensitive information to the cloud servers. Passwords for local devices (e.g., laptops, tablets, smartphones) and cloud service providers must be updated and renewed every 30 days as recommended by many security experts ${ }^{[12]}$. Judicious use of LastPass ${ }^{\circledR}$ and Carbonite ${ }^{\circledR}$ to facilitate frequent and automatic password changes and document uploads is advised by the authors. Researchers also should verify the reputations of the cloud service providers with respect to procedures for backup, restoration, and disaster recovery as well as for ongoing data security maintenance. In fact, this may need to be addressed in their proposal when Institutional Review Board (IRB) approval is sought. 
Lack of applications for researcher use has come to the attention of those who conduct research. For example, an Android market search for biostatistics packages yielded no application comparable to commonly-used comprehensive statistical packages such as SPSS ${ }^{\circledR}$, Stata ${ }^{\circledR}$, or STATISTICA ${ }^{\circledR}$. It may be necessary for researchers to prepare data files in Excel, then import them via WiFi or iTunes file sharing. This could be a significant hindrance to those who need to enter data quickly into a secure environment. For example, the nurse researcher who interviews limited-access subjects, such as those who are incarcerated, may have only a narrow window of opportunity or restricted tools to capture the interaction. Pending the availability of the proper applications, rapid-access cloud computing could secure these data.

The remaining disadvantages of cloud computing, especially when tablets are used, concern usability. End users cannot easily migrate data between cloud service providers. The amount of data storage capacity and the measurement of usage is at the discretion of the provider. Researchers are accustomed to typing their data, but the virtual touch screen of the tablet is not designed for long messages, extensive data entry, or manipulation of long documents. The addition of a battery-operated wireless mechanical keyboard can overcome this but adds a device to the repertoire.

\subsection{An Overview of tablet computing}

A complete mobile computer, a tablet is larger than a mobile phone or personal digital assistant (PDA) and contains an integrated flat-touch screen. Usually operated by an onscreen virtual keyboard, a passive stylus, or a digital pen, tablet personal computers (PCs) are essentially laptop computers without the external keyboard or other peripherals. Often running on such operating systems as Windows, Linux, or Android, the most popular of these small, lightweight devices are Apple's iPad 2 and Motorola's Xoom. Dating back to the 1950s, the first publicly-demonstrated system that used a tablet and handwriting text recognition, instead of a keyboard, was invented by Tom Dimond (Styalator electronic tablet) and reinvented in the early 1960s by both RAND (RAND Tablet) and Xerox (Dynabook) ${ }^{[13]}$.

Electronic clipboards were popularized in television and film when the crew of Star Trek carried large wedge-shaped electronic boards (1966) and when Stanley Kubrik's flat-screen tablet device played streaming video in 2001: A Space Odyssey. Since that time, the use of devices such as a pen computer or a touch-screen computer has had limited exposure until the 2010s, when Apple unveiled the iPad, which runs Apple iOS, and Motorola released the Xoom Honeycomb, which runs Android 3.0. Today, these devices are owned by people with any skill level, from casual users, who primarily want storage for family pictures, to white-collar professionals, who crave professional document portability.

Although documents can be downloaded and saved, this is not the purpose of the tablet, partly because large-scale document revision or other management is less feasible on it. The tablet's ability to open instantly complements its reliable and fast access to documents saved and shared in a cloud, obviating the need for a heavy and cumbersome traditional laptop. Professional users of tablets can word process and manipulate data sets through such applications as Quickoffice Pro ${ }^{\circledR}$ or Documents To Go®. Tablets have all the application capacity of a smart phone and, as such, can serve as a recorder, alarm clock, PDA, camera, mp3 player, and much more. Most tablets lack an external input port, thus making document storage on a cloud more attractive. Yet, the researcher who relies on the display of electronic media through use of a DVD as a treatment might find this feature to be a barrier. We advocate the tablet as a complement to smart phones and as a mobile replacement for standard desktop computers, particularly when international face-to-face encounters occur.

\subsection{What cloud and tablet computing mean in a wikinomics future}

Cloud computing provides end users with the opportunity to store, access, share, and consume information via the Internet, without the need to be concerned with its physical location or the computer expertise of the end user. Cloud computing is a service, not a product, whereby resources are shared and "open." In healthcare, there is a move away from an all-encompassing model of IT oversight on premises to organizations' running applications on hardware located and managed off-site through a shared service arrangement ${ }^{[14]}$. Thus, SaaS will enable healthcare organizations to use the applications and infrastructure of another organization, thereby reducing operational expenses and raising productivity through reduced opportunity costs. 
Complemented by the use of minicomputers, personal computers, and more recently, tablet computers, cloud computing provides an infrastructure that can be scaled by end users to meet the increasingly complex demands of IT agendas in healthcare. Tablet computers, among the fastest mobile devices, are particularly well suited to enable rapid-fire access to data and documents in clouds. Further, tablet capabilities such as follow-me printing, persistent-state roaming, storage for Single-Sign-On (SSO), and rapid-access video conferencing enhance the productivity and lessen tedium for nurse researchers and other healthcare professionals.

\subsection{Cloud and tablet computing in nursing research}

Maximum efficiency is derived through the joint use of cloud and tablet computing. Each was designed with the mobile user in mind. Nevertheless, as autonomous professionals, those in healthcare, including nurse researchers, are generally not known for their mobile computer competencies. Notably, their professional competence and confidence in discharging their duties may be challenged when new work processes are introduced ${ }^{[15]}$. To use mobile computing solutions at the point of care or of data collection, the nurse researcher will need to develop informatics competencies such as document storage and retrieval involving clouds. The re-engineering of work processes, through the use of e-health operational applications, represents a radical change in healthcare ${ }^{[15]}$. In Figure 5 below, the primary venue for work processes (vertical axis) is shown against a timeline to adoption on the horizontal axis, patterned after the Diffusion of Innovation Model by Rogers ${ }^{[16]}$.

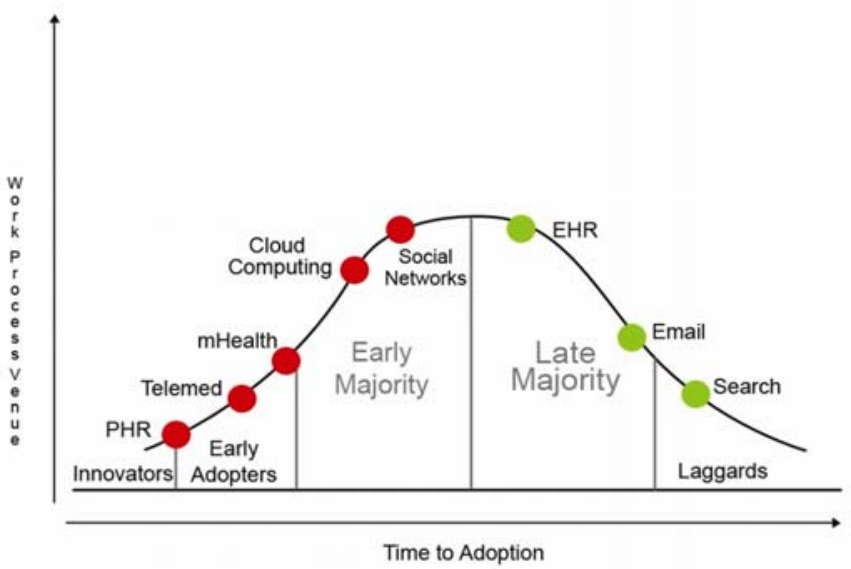

Figure 5. The primary venue for work processes

Based on this figure, we see that the use of cloud computing likely falls in the early majority category of innovation adoption. A user in this category is a willing follower, who, shown the value of a technological innovation, such as cloud computing, will use a product or service fairly readily. In regard to the change in work processes, this early majority adopter has a longer diffusion time, which, according to change leadership theorists, is the opportunity afforded to refreeze or solidify new habits and practices ${ }^{[16,17]}$. The transition from silo (isolated) desktop computer use, whereby people work primarily through use of email and attachments (late majority), to the more agile cloud/tablet computing arrangement can be considered one of early majority use.

The need for this transition is accentuated when one takes into account the massive shift from on-site computing under SaaS to off-site and/or offshore storage and service, which eliminates or limits the need for local IT personnel. Professionals such as nurse researchers must become competent informaticians to execute the operations of their investigation. Even if those work processes are delegated to a research assistant, the researcher must be able to confirm the security and privacy of the datasets used in each step of the work plan, which is likely to be done with significantly less on-site help. Nurse researchers who serve as their own primary data-entry and data-retrieval specialists must be prepared to embrace these changes in work flow in the Wikinomics future. 


\section{Conclusion}

Little or nothing in the literature addresses the competencies needed by the nurse researcher wishing to gather, archive and analyze data while mobile. The research process can be facilitated by the use of the selected tools discussed here, especially in the data collection and early analysis phases. As nurse researchers acquire the necessary skills to use these tools, it is likely that the collaboration on shared projects will be enhanced, particularly in the research development stage. Preparation for the role of visiting scholar abroad should include the selection of digital tools appropriate to the research method and the preferences of the research team, including the features and type of cloud.

\section{Acknowledgement}

The authors acknowledge the assistance of Dr. Nanda Ganesan of California State University, Los Angeles (Professor, Information Systems) during the manuscript revision phase.

\section{References}

[1] Tapscott, D and Williams, AD. Wikinomics. In Wikinomics: How Mass Collaboration Changes Everything. D. Tapscott and A.D. Williams, eds. New York: Penguin Group Inc. 2008; 7-33.

[2] O’Reilly T. 2004. The Architecture of Participation [Internet]. Available from: http://oreilly.com/pub/a/oreilly/tim/articles/architecture_of_participation.html (31 December 2011, date last accessed).

[3] Jankowski NW. The contours and challenges of e-research. In E-Research: Transformation in Scholarly Practice. N.W. Jankowski, ed. New York: Routledge. 2009; 1-20.

[4] Halfpenny P, Proctor R. The e-Social Science research agenda. Royal Phil Trans R Soc A. 2010; 368: 3761. PMid:20643675 http://dx.doi.org/10.1098/rsta.2010.0154

[5] NeSC. http://www.nesc.ac.uk/nesc/define.html (31 December 2011, date last accessed).

[6] Wang T, Chien A. The influences of technology development on economic performance-the example of ASEAN countries. Technovation. 2007; 27 (8): 471-488. http://dx.doi.org/10.1016/j.technovation.2007.04.006

[7] Kroch E, Duan M, Silow-Carrol S, Meyer JA. Hospital Performance Improvement: Trends in Quality and Efficiency-A quantitative Analysis of Performance Improvement in U. S. hospitals. 2007. Available from: http://www.commonwealthfund.org/Publications/Fund-Reports/2007/Apr/Hospital-Performance-Improvement--Trends-in-Qualit y-and-Efficiency-A-Quantitative-Analysis-of-Perfor.aspx (31 December, 2011, date last accessed).

[8] Parkhill DF. Modern computer technology. In The Challenge of Computer Utility. D.F. Parkhill, ed. Boston: Addison-Wesley Publishing Co. 1966; 1-20.

[9] Campbell AT, Eisenman SB, Lane ND, Miluzzo E, Peterson RA, Lu H. The rise of people-centric sensing. Internet computing, IEEE. 2008; 2(4): 12-21.

[10] Younge AJ, von Laszewski G, Wang L, Lopez-Alarcon S, Carithers W. Efficient resource management for cloud computing. In Proceedings of International Conference on Green Computing. 15-18 August 2010; Chicago: 357-364. http://dx.doi.org/10.1109/GREENCOMP.2010.5598294

[11] Piette JD, Mendoza-Avelares MO, Ganser M, Mohamed M, Marinec N, Krishnan, S. A preliminary study of a cloud-computing model for chronic illness self-care support in an underdeveloped country. Am J Prev Med. 2011; 40: 629-632. PMid:21565655 http://dx.doi.org/10.1016/j.amepre.2011.02.014

[12] Furnell S. An assessment of website password practices. Computers and Security. 2007; 26(7-8): 445-451. http://dx.doi.org/10.1016/j.cose.2007.09.001

[13] Atkinson PA. A bitter pill to swallow: the rise and fall of the tablet computer. Design Issues. 2008; 24(4): 3-25. http://dx.doi.org/10.1162/desi.2008.24.4.3

[14] Glaser J. Cloud computing can simplify HIT infrastructure management. Healthc Financ Manage. 2011; 65(8): 52-5.

[15] Rigby M. Essential prerequisites to the safe and effective widespread roll-out of e-working in health care. Medical Informatics. 2005; 75: 138-147.

[16] Rogers EM. A prospective and retrospective look at the diffusion model. J Health Commun Suppl. 2004; 9(1): 13-19. PMid:14960401 http://dx.doi.org/10.1080/10810730490271449

[17] Greenhaigh T, Stramer K, Bratan T, Byrne E, Mohammad Y, Russel J. Introduction of shared electronic records: multi-site case study using diffusion of innovation theory. BMJ. 2008; 337: a1786. 